\title{
Generating Non-Gaussian Adiabatic Fluctuations from Inflation
}

\author{
D.S. Salopek ${ }^{1}$ \\ Department of Physics 83 Astronomy \\ 6224 Agricultural Road, University of British Columbia \\ Vancouver, Canada V6T $1 Z 1$ \\ To appear in \\ Proceedings of COSMO-98 \\ International Workshop on Particle Physics and the Early Universe \\ Monterey, California, Nov. 15-20, 1998 \\ Ed. D. Caldwell (Published by American Institute of Physics)
}

\begin{abstract}
As the quality of cosmological data continue to improve, it is natural to test the statistics of primordial fluctuations: are they Gaussian or non-Gaussian? I review a model which generates non-Gaussian adiabatic fluctuations from inflation. Current investigations suggest that there may possibly be a non-Gaussian signal in large angle cosmic microwave background anisotropy data. Statistics of microwave anisotropies could thus serve as a powerful probe of the very early Universe.
\end{abstract}

\section{INTRODUCTION}

The inflationary expansion of the very early universe acts like a microscope that magnifies short scale quantum fluctuations to encompass our entire observable Universe. In a sense, microwave background anisotropy maps such as the COBE DMR maps provide an image of the vacuum state that arose from inflation. If the field interactions during the inflationary epoch are linear, then one expects that the statistics of vacuum fluctuations will be Gaussian - this is the situation that most researchers are hoping for. However, there are indeed models where significant

1) E-mail: dsscosmos@yahoo.com

2) Current mailing address: Space-Time Institute, 14915-105 Avenue, Edmonton, Alberta, Canada T5P 4M2 
nonlinearities arise which would produce a non-Gaussian signal. Here, I will review such an inflation model which produces non-Gaussian adiabatic fluctuations [1].

By considering 3-point correlations of spherical harmonic amplitudes in COBE DMR maps, Ferreira et al [2] have found evidence of a non-Gaussian signal (see also Magueijo [3]). They point out a non-Gaussian signal associated with the $\ell=16$ multi-pole of a spherical harmonic expansion. They are not able to associate the signal with any known foreground emission, although they are concerned that the power takes a mysterious dip at this same value of $\ell$. Their work requires further investigation.

The computation of nonlinear effects during inflation is a challenging problem. However, nonlinearities are essential to the formulation of the inflationary scenario. For example, at the end of inflation, the inflaton must transfer its energy to a bath of radiation and matter; nonlinearities must thus be significant. The 'heating' of the Universe is an interesting problem that is currently under investigation (see, e.g., Kofman [9]). The computation of nonlinear effects during the slow-roll period of inflation (e.g., before heating of the Universe) faces two severe theoretical obstacles:

(1) The complete field theory that encompasses inflation is not known. String theory may provide the required framework although this remains to be seen.

(2) Quantum gravity effects are important during inflation. For example, it is generally agreed that the quantum generation of tensor modes (gravitational waves) during inflation is a quantum gravity effect although it is only a 'linear' effect.

Soon after the COBE detection in 1992, it was pointed out by Salopek [6] and Davis et al [7] and subsequently verified by many others that tensor modes from inflation could indeed contribute significantly to COBE's signal. In particular, Salopek [6] pointed out that at most 50\% of COBE's signal could be attributed to tensor modes arising from power-law inflation otherwise there would not be enough fluctuations to account for the observed clustering of galaxies. (For a careful analysis of how current cosmological data restricts tensor modes, consult Zibin et al [8]; they confirm the above $50 \%$ bound that was deduced over five years earlier. In this conference, Kinney [9] explains how the measurement of polarization by future microwave background anisotropy experiments will help to determine the contribution of tensor modes more accurately.)

In order to bypass severe problems associated with nonlinear quantum effects of gravity, Stewart and Parry and I advocate solving the Hamilton-Jacobi equation for general relativity [10] which provides a nonlinear semiclassical description of gravity interacting with matter. We attempt to imitate the historical development of the theory of atomic spectra. Before the development of the quantum theory in 1926, the semiclassical theory of Bohr and Sommerfeld provided a useful although imperfect description of various atoms.

The non-Gaussian model described below employs several unique features of the Hamilton-Jacobi equation. It has a distinct observational signature: it produces a non-Gaussian distribution for cosmic microwave temperature anisotropies. This model is constrained by the analysis of DMR maps by Kogut et al [4] and Ferreira et al [2]. Typically each non-Gaussian model produces a specific signature which 
allows one to discriminate among the different models. This property provides a powerful probe of the very early Universe.

\section{HAMILTON-JACOBI EQUATION FOR COSMOLOGY}

In a Hamilton-Jacobi (HJ) formulation, there are just two laws that govern the inflationary epoch:

$$
\mathcal{H}(x)=0, \quad \mathcal{H}_{i}(x)=0 .
$$

The first legislates that the total energy density vanishes at every point in the Universe, whereas the second legislates that the total momentum density vanishes at every point. In fact, it is a great accomplishment that one may write two mathematical laws that govern the very early Universe. Explicitly, the energy and momentum densities may be written in terms of the scalar fields, $\phi_{a}$, the 3 metric, $\gamma_{i j}$, and their respective momenta, $\pi^{\phi_{a}}, \pi^{i j}$ :

$$
\begin{aligned}
\mathcal{H}= & \kappa \gamma^{-1 / 2}\left[2 \gamma_{i k} \gamma_{j l}-\gamma_{i j} \gamma_{k l}\right] \pi^{i j} \pi^{k l}+\frac{\kappa}{2} \sum_{a} \gamma^{-1 / 2}\left(\pi^{\phi_{a}}\right)^{2}+\kappa \gamma^{1 / 2} V\left(\phi_{a}\right)+ \\
& \left\{-\frac{1}{2 \kappa} \gamma^{1 / 2} R+\frac{1}{2 \kappa} \gamma^{1 / 2} \sum_{a} \phi_{a \mid i} \phi_{a}{ }^{\mid i}\right\}, \\
\mathcal{H}_{i}= & -2\left(\gamma_{i j} \pi^{j l}\right)_{, l}+\pi^{k l} \gamma_{k l, i}+\sum_{a} \pi^{\phi_{a}} \phi_{a, i},
\end{aligned}
$$

where $\kappa=8 \pi G$ and $R$ denotes the Ricci curvature of the 3-metric. The object of principal interest is the generating functional $\mathcal{S} \equiv \mathcal{S}\left[\gamma_{i j}(x), \phi_{a}(x)\right]$ which assigns a number (in general complex) to each field configuration $\phi_{a}(x)$ on a space-like hypersurface described by the 3 -metric $\gamma_{i j}(x)$. The momenta are given by functional derivatives of the generating functional:

$$
\pi^{\phi_{a}}=\frac{\delta \mathcal{S}}{\delta \phi_{a}}, \quad \pi^{i j}=\frac{\delta \mathcal{S}}{\delta \gamma_{i j}} .
$$

The constraints equations (1) are self-contained equations for the generating functional $\mathcal{S}$, which is just the 'phase' of the wavefunctional in the semiclassical limit.

\section{Strong-Coupling Limit: $G \rightarrow \infty$}

To solve for the generating functional, $\mathcal{S}$, one must resort to approximation methods. In the limit that Newton's constant $G=\kappa /(8 \pi)$ is large, one may safely neglect the terms appearing within the braces \{\} of eq.(2a). The resulting system, Strongly-Coupled Gravity and Matter, is exactly solvable [11]. Its simplest solution is of the form 


$$
\mathcal{S}=-2 \int d^{3} x \gamma^{1 / 2} H\left(\phi_{a}\right)
$$

where the function $H \equiv H\left(\phi_{a}\right)$ is a function of the scalar fields and it satisfies the separated Hamilton-Jacobi equation (SHJE):

$$
H^{2}=\frac{2}{3} \sum_{a}\left(\frac{\partial H}{\partial \phi_{a}}\right)^{2}+\frac{1}{3} V\left(\phi_{a}\right) .
$$

Explicit general solutions of the SHJE exist for potentials of the exponential type

$$
V(\phi)=V_{0} \exp \left(\sum_{a} B_{a} \phi_{a}\right) ;
$$

that is when $\ln V(\phi)$ is linear, $H$ is exactly solvable. More complicated potentials are obtained by gluing together different exponential potentials together [1].

\section{NON-GAUSSIAN ADIABATIC FLUCTUATIONS}

For inflation with two scalar fields, non-Gaussian fluctuations arise in a model where one glues continuously three exponential potentials of the type given by eq.(4). Such a model is illustrated in Fig.1. The resulting distribution in large angle temperature anisotropies is shown in Fig. 2. It is strongly non-Gaussian. The impact of similar models on the distribution of galaxies has been investigated by Fan and Bardeen [12] as well as Moscardini et al [13].

The challenge in the future is to see if the parameters of the model may be adjusted to fit the non-Gaussian signal of Ferreira et al [2] and yet be consistent with the limits of Kogut et al [4].

\section{DISCUSSION}

Recent analysis suggests that the COBE DMR map may have a non-Gaussian signal [2]. It is definitely possible to construct inflation models which produce non-Gaussian fluctuations [1]. By studying the statistics of microwave background fluctuations, one hopes to develop a powerful probe of the very early Universe. The situation should improve dramatically when the next generation of microwave background anisotropy satellites such as MAP and PLANCK are launched.

\section{REFERENCES}

1. Salopek, D.S., Phys. Rev. D45, 1139 (1992).

2. Ferreira, P.G., Magueijo, J., and Gorski, K.M., astro-ph/9803256 (1998).

3. Magueijo, J., these proceedings (1999). 
4. Kogut, A., Banday, A.J., Bennett, C.L., Gorski, K.M., Hinshaw, G., Smoot, G.F., and Wright, E.L., Astrophy. J. 464, L5 (1996); ibid L29 (1996).

5. Kofman, L., these proceedings (1999).

6. Salopek, D.S. Phys. Rev. Lett., 69, 3602 (1992).

7. Davis, R.L. et al, Phys. Rev. Lett. 69, 1856 (1992).

8. Zibin, J.P., Scott, D., and White, M., University of British Columbia preprint (1999).

9. Kinney, W. these proceedings (1999).

10. Parry, J., Salopek, D.S. and Stewart, J.M., Phys. Rev. D49, 2872 (1994).

11. Salopek, D.S., Class. Quant. Grav. 15, 1185 (1998); ibid 16, 299 (1999).

12. Fan, Z.H., and Bardeen J.M., Predictions of a Non-Gaussian Model for Large Scale Structure, UW-PT-92-11, KEK library scanned preprint: 9207442 (1992).

13. Moscardini, L., Borgani, S., Coles, P., Lucchin F., Matarrese S., Messina, A., and Plionis, M., Astrophys. J. Lett. 413, 55 (1993).

\section{FIGURE 1}

Non-Gaussian fluctuations may be generated during inflation when two scalar fields pass over a sharp but continuous feature in their potential. The light solid curves are lines of uniform scalar field potential; the heavy lines are the boundaries between the 3 regions where $\ln V$ is linear. By choosing the initial conditions, the scalar field trajectories (broken lines) which start in the lower left hand corner may pass sufficiently near the origin, and nonlinear effects become important.

\section{FIGURE 2}

For the model described in Fig. 1, a very significant non-Gaussian histogram in large angle cosmic microwave maps is produced. This extreme distribution is shown for illustrative purposes. By adjusting the input parameters, one may arbitrarily adjust the height and placement of the left peak. For comparison, the dark curve is the best fit Gaussian. 


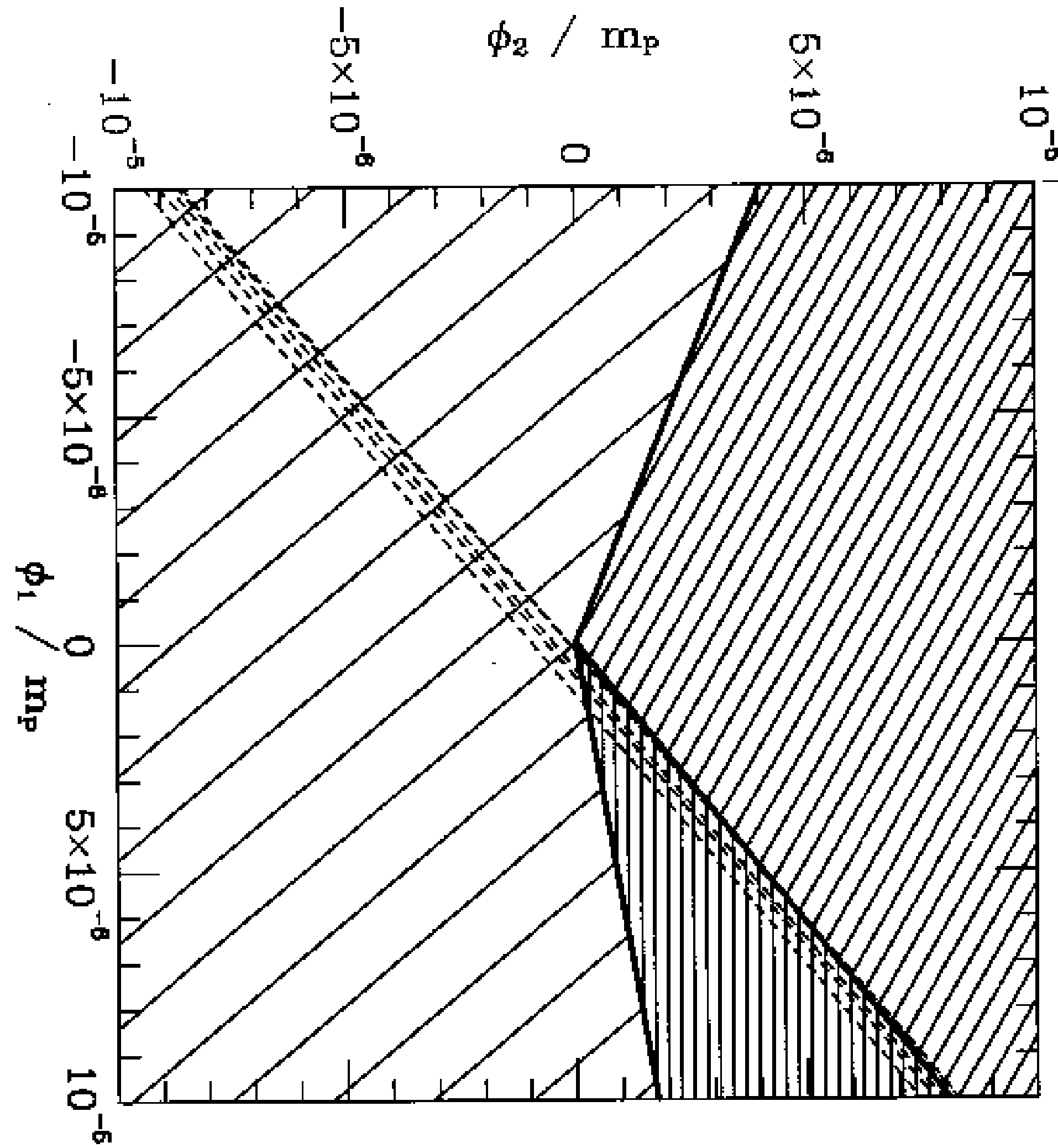

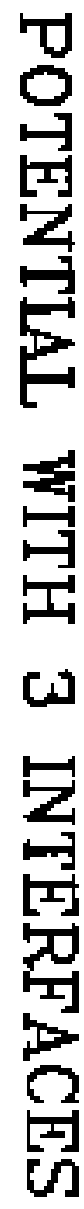




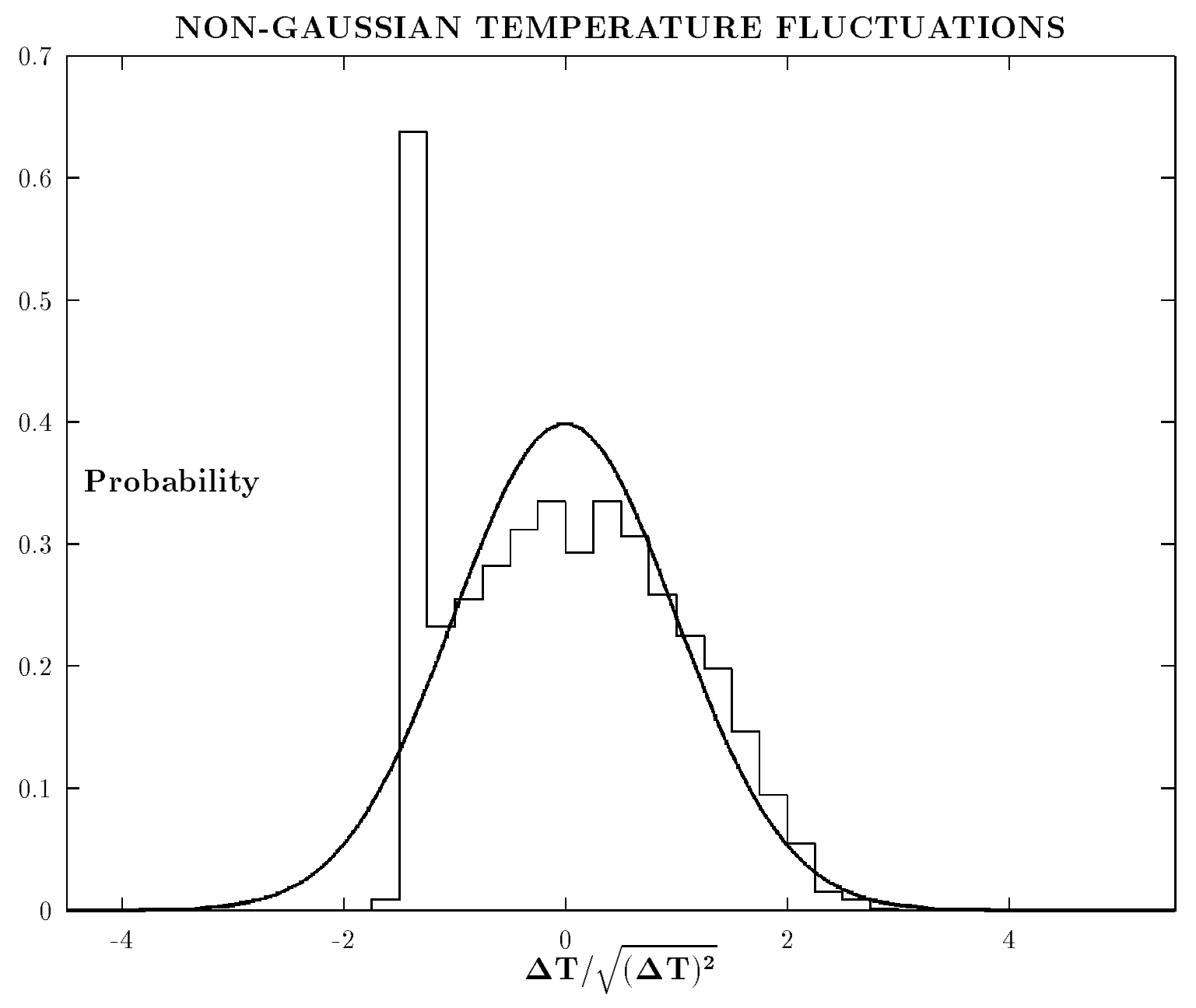

